\title{
Aluminum foil reinforced by carbon nanotubes
}

\author{
A. V. Alekseev ${ }^{1}$, M. R. Predtechenskiy ${ }^{2}$ \\ ${ }^{1}$ International Science Centre of Thermal physics and Energetics, Novosibirsk, Russia \\ ${ }^{2}$ Institute of Thermalphysics, Novosibirsk, Russia \\ artem.alekseev@ocsial.com, predtechensky.mr@ocsial.com
}

PACS 81.05.Ni, 81.20.Ev, 81.40.Ef

DOI 10.17586/2220-8054-2016-7-1-185-189

In our research, the method of manufacturing an Al-carbon nanotube (CNT) composite by hot pressing and cold rolling was attempted. The addition of one percent of multi-walled carbon nanotubes synthesized by OCSiAl provides a significant increase in the ultimate tensile strength of aluminum. The tensile strength of the obtained composite material is at the tensile strength level of medium-strength aluminum alloys.

Keywords: carbon nanotubes, aluminum, composite, hot pressing, cold rolling.

Received: 20 November 2015

\section{Introduction}

The addition of carbon nanotubes can significantly improve the mechanical properties of aluminum and its alloys [1-3]. At the present moment, powder metallurgy methods are mostly used to produce aluminum-based composites reinforced by carbon nanotubes (CNT). These methods include the manufacturing of a powder compact followed by its hot deformation [4]. Compacts are produced by spark plasma sintering [4], cold pressing followed by sintering [5], explosive compacting [6], hot isostatic pressing [7] and hot pressing [8]. The final operation of composite manufacturing is hot extrusion [4] or hot rolling [9]. There are articles in which the authors successfully combined powder compacting and hot extrusion, and thus realized the spark plasma extrusion method [10].

In this work, aluminum composite reinforced by CNT was produced via a hot pressing and final cold rolling operation.

\section{Experimental procedure}

For powder mixtures, aluminum powder (preparation standard PA-1) (GOST6058-73) and multiwalled carbon nanotubes (MWCNT) manufactured by OCSiAl (see Fig. 1) were used. Powder mixtures containing $1 \mathrm{wt} . \%$ CNT were made by high energy milling in planetary ball mill AGO-2S. Thereafter, powder mixtures were compacted by hot pressing at $50 \mathrm{MPa}$ at a temperature of $600-670{ }^{\circ} \mathrm{C}$ under argon. During cold rolling, $10 \%$ deformation for each pass was produced. Approximately $10-15$ passes were made to obtain each sample of foil. Part of the foil samples were then annealed at $350{ }^{\circ} \mathrm{C}$ for $120 \mathrm{~min}$.

For comparison, we made aluminum foil with additions of $1 \mathrm{wt} . \%$ of carbon powder VulkanXC72 and foil without any additives. These foil samples were made in the same way as those with CNT additives. Analysis of composite structure was conducted by scanning electron microscope LEO1430-VP. A Bruker d8 Advance diffractometer with parallel geometry and $\mathrm{CuK}_{\alpha 1}=1.5406 \AA ; \mathrm{Cu} \mathrm{K}_{\alpha 2}=1.54439 \AA$ radiation was used for carbide detection.

Foil samples with $100 \mu \mathrm{m}$ thickness were cut to obtain samples for tensile strength determinations. Tensile tests were conducted at room temperature by a servo- hydraulic testing machine RPM-50U. 


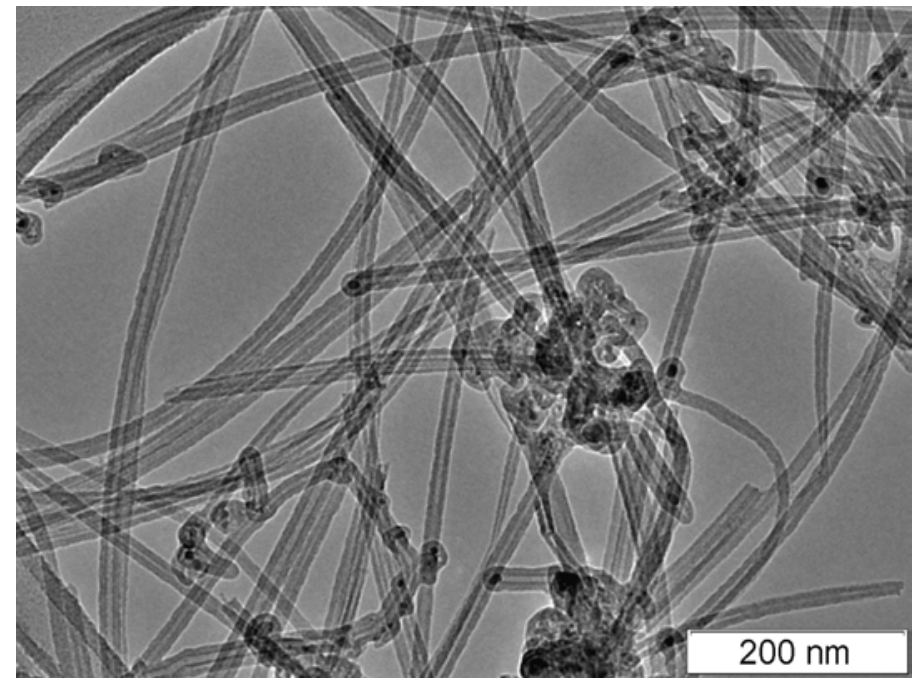

(a)

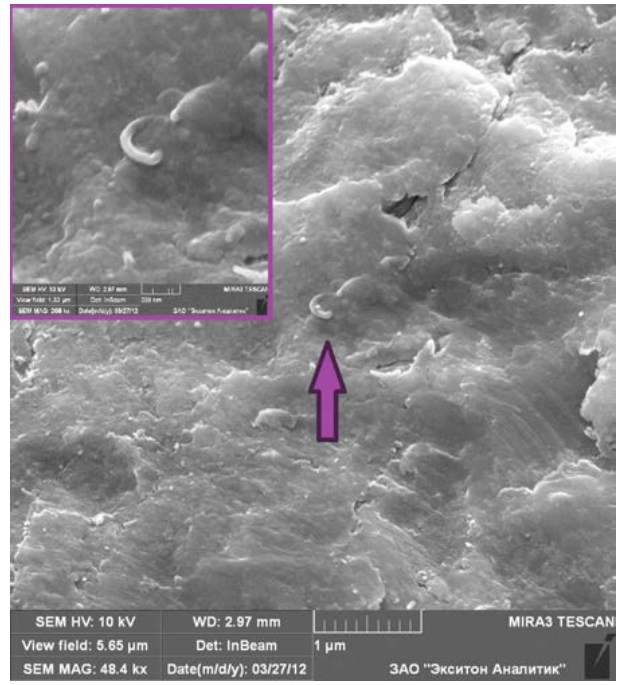

(b)

FIG. 1. TEM photographs of MWCNT

\section{Results and discussion}

SEM photographs of powder mixtures are shown in Fig. 2. The powder mixture containing CNT consisted of mostly spherically-shaped particles. The diameter of powder particles is typically $250-270 \mu \mathrm{m}$ and rarely exceeds $900 \mu \mathrm{m}$. Detailed analysis of powder surface by high resolution SEM did not reveal CNT agglomerates but showed separated CNT embedded in the metal particles' surfaces.

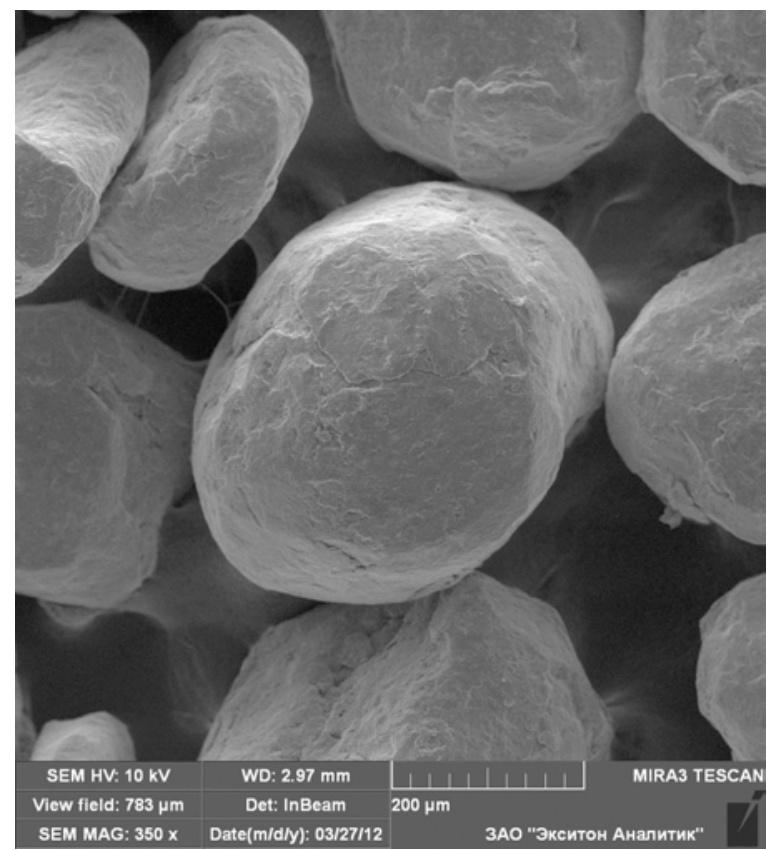

(a)

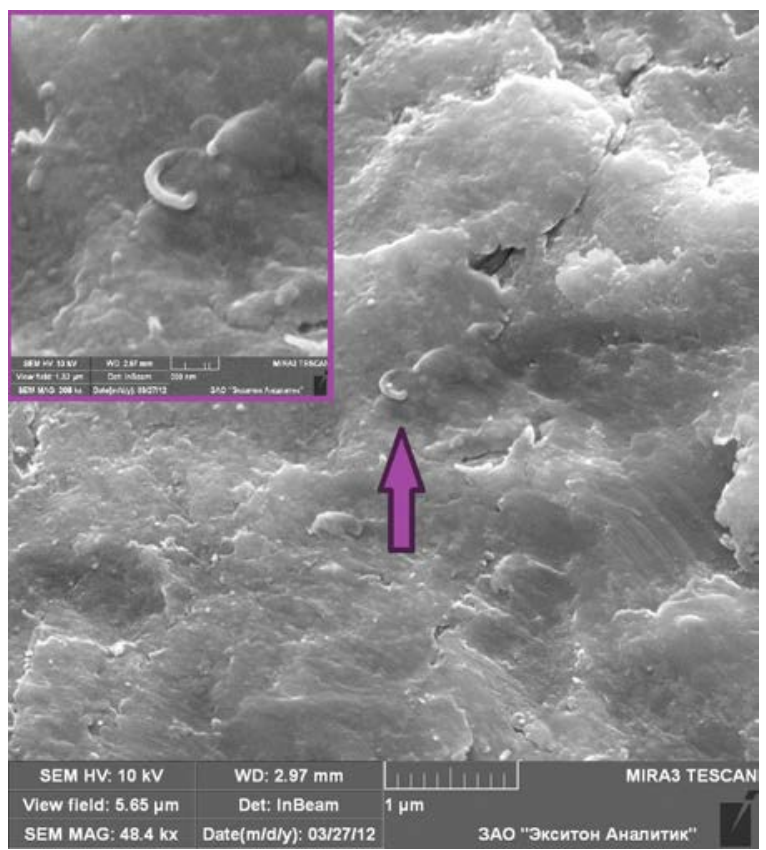

(b)

FIG. 2. SEM photographs of powder mixtures containing $1 \mathrm{wt} . \% \mathrm{CNT}$

X-ray diffraction spectra of powder mixture containing CNT, and aluminum foil containing CNT are presented in Fig. 3. It is well known that the presence of a large amount of 
aluminum carbides decreases the mechanical properties of an aluminum-CNT composite [12]. Consequently, it is preferable to minimize aluminum carbide formation during the composite manufacturing process in order to produce materials with improved mechanical properties.

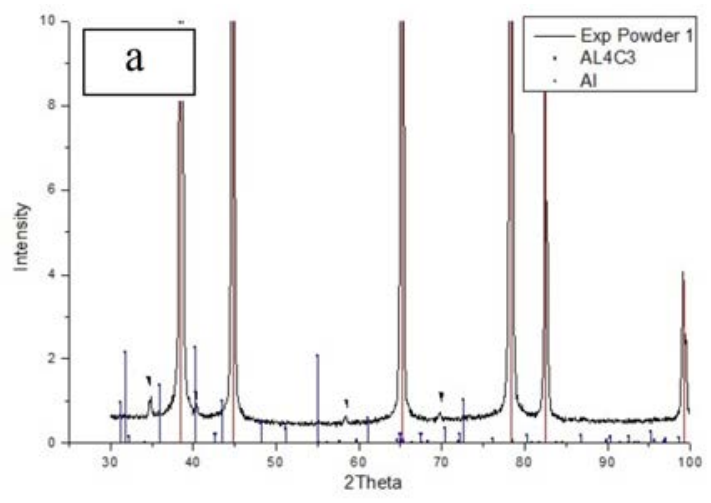

(a)

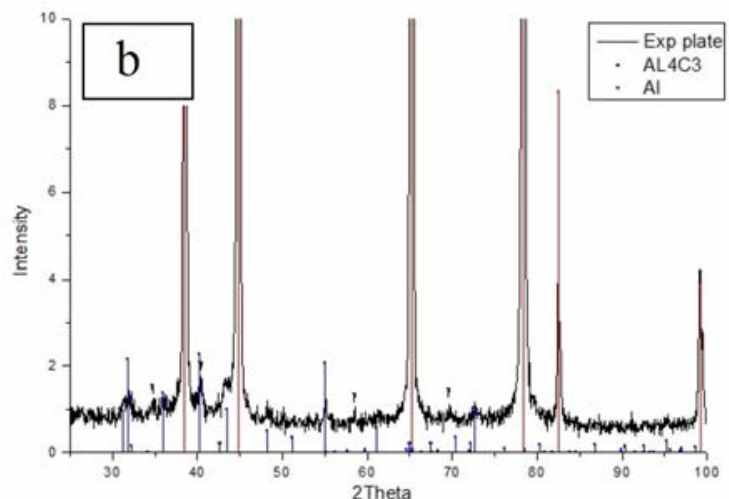

(b)

FIG. 3. XRD spectra of powder mixture containing 1 wt.\% CNT (a) and composite foil $\mathrm{Al}-1$ wt.\% CNT (b)

Conversely, the existence of a minute amount of aluminum carbide, in the form of nanosized particles positioned on the CNT-aluminum interfaces can increase the adhesion between CNT and metal matrix [1, 2, 13]. Since aluminum carbide can appear not only during high energy powder mixing [11] but also during the hot pressing of powder mixtures, it is necessary to control its quantity at each step of the composite manufacturing process. As shown in Fig. 3, no aluminum carbide is present in the aluminum-CNT powder mixtures. Only trace amounts of aluminum carbide was shown to be present in our composite foil.

SEM photographs of fracture surfaces from hot pressed powder compacts are shown in Fig. 4. Structures of both compacts are homogeneous. One can see that the length of protruded CNT is not more than $1 / 10$ of the initial CNT length. This indicates good adhesion between the CNT and the metal [13-16].

Table 1 contains the results of the tensile strength test for cold rolled aluminum foils. The tensile strength increase for composite foil can be caused not only by CNT addition, but also by amorphous carbon powder formation during CNT collisions in a high energy ball mill. In this sense, it is more correct to compare composite foil and control samples reinforced by amorphous carbon powder. For this reason, we made two types of control samples. The first one contains amorphous carbon powder additives and the second one has no carbon additives.

TABLE 1. Tensile test results

\begin{tabular}{|c|c|c|}
\hline Sample number & Powder mixture type & Tensile strength, MPa \\
\hline 1 & $\mathrm{Al}+1 \% \mathrm{CNT}$ & 441 \\
\hline 2 & $\mathrm{Al}+1 \%$ carbon powder & 360 \\
\hline 3 & $\mathrm{Al}$ & 357 \\
\hline
\end{tabular}

From Table 1, it is clear that foil samples that contain powder of amorphous carbon have strength similar to that of control foil samples without any additives. 


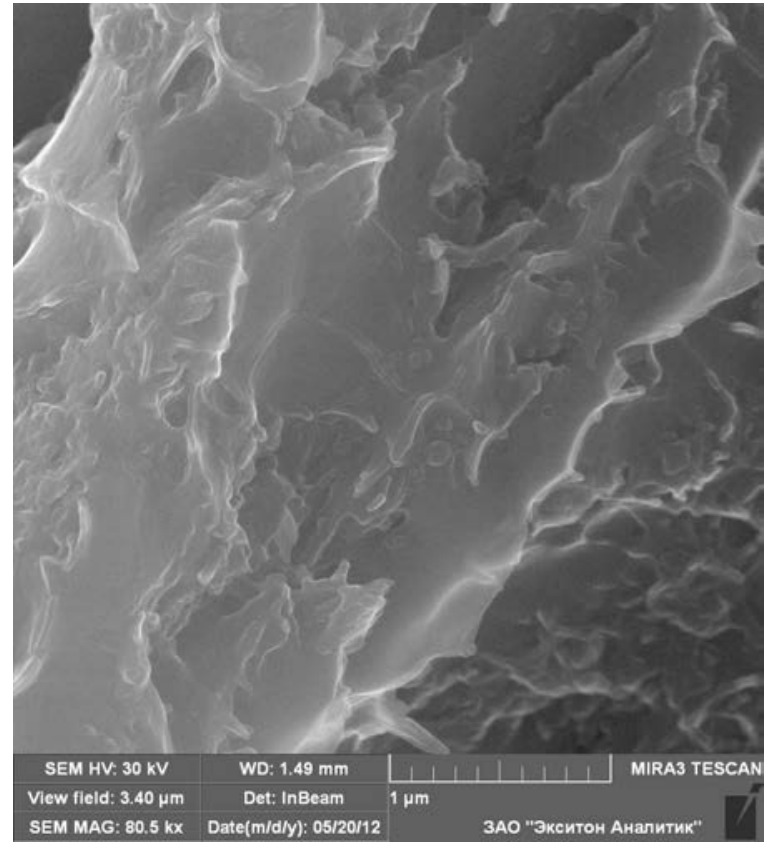

(a)

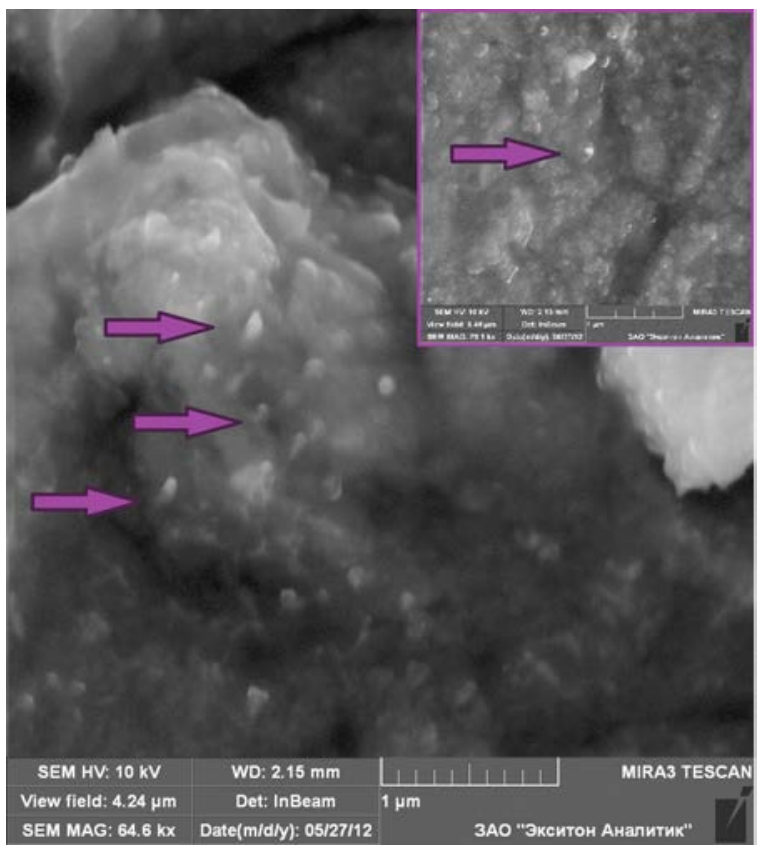

(b)

FIG. 4. SEM photographs of hot pressed powder compact with additives of carbon powder (a) and CNT (b)

The tensile strength of aluminum foil with $1 \%$ wt. CNT added was increased by $22 \%$ relative to pure aluminum foil. We can conclude that strength increase is caused only by influence of CNT. All of samples had low plasticity, which may be explained by the high degree of cold working.

Annealed samples of foil with 1 wt.\% CNT also had high tensile strength. Comparative diagram of our composite foil and standard aluminum foil GOST618-73 are shown in Fig. 5. These data clearly show that our composite foil surpasses the standard aluminum foil strength by a factor of 3 under both annealed and cold worked conditions.

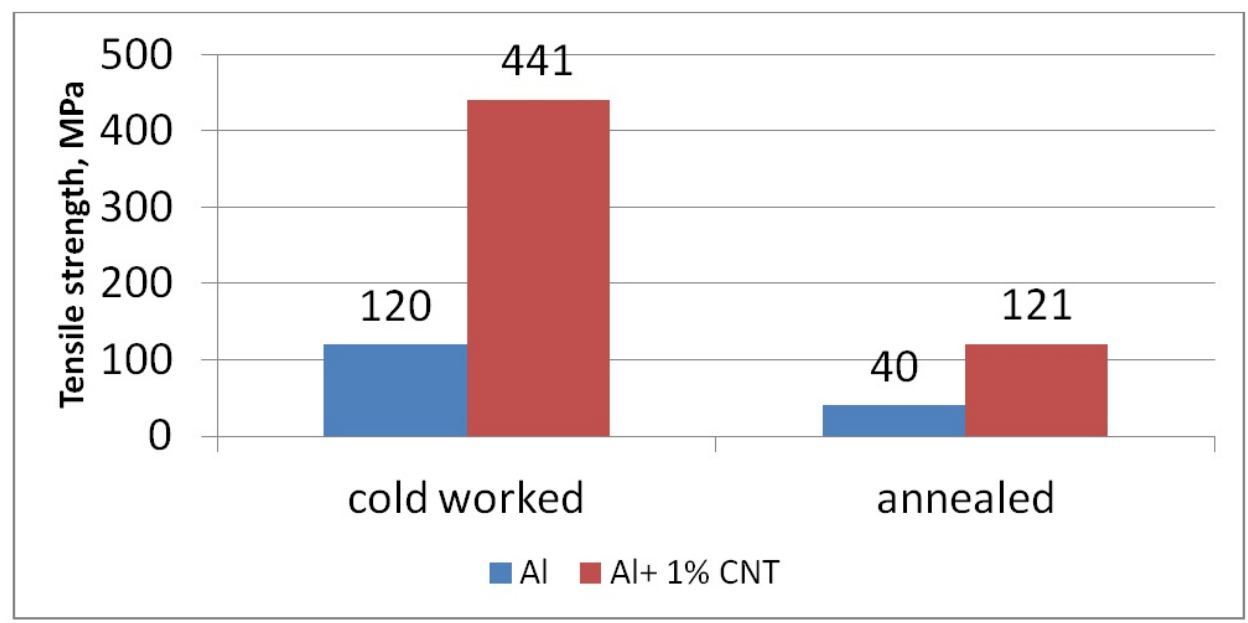

FIG. 5. Comparative diagram of composite foil tensile strength and standard foil tensile strength 


\section{Conclusion}

Composite foil reinforced by CNT was made by hot pressing and subsequent cold rolling. The addition of $1 \mathrm{wt} . \%$ of MWCNT manufactured by OCSiAl provided an increase in the tensile strength of aluminum foil by $22 \%$. Our composite has a tensile strength comparable to that of medium strength aluminum alloys and is 3 -fold greater than standard aluminum foil.

\section{References}

[1] Kwon H., Park D.H., Silvain J.F., Kawasaki A. Investigation of carbon nanotube reinforced aluminum matrix composite materials. Composites Sci. Technol., 2010, 70(3), P. 546-550.

[2] Bakshi S.R., Agarwal A. An analysis of the factors affecting strengthening in carbon nanotube reinforced aluminum composites. Carbon, 2011, 49(2), P. 533-544.

[3] Choi H.J., Shin J.H., Bae D.H. Grain size effect on the strengthening behavior of aluminum-based composites containing multi-walled carbon nanotubes. Composites Sci. Technol., 2011, 71, P. 1699-1705.

[4] Liao Jin-zhi, Tan Ming-Jen, Sridhar I. Spark plasma sintered multi-wall carbon nanotube reinforced aluminum matrix composites. Materials \& Design, 2010, 31(1), P. 96-100.

[5] Singhal S.K., Pasricha R., Jangra M., Chahal R., Teotia S., Mathur R.B. Carbon nanotubes: Amino functionalization and its application in the fabrication of Al-matrix composites. Powder Technol., 2012, 215-216, P. 254-263.

[6] Salas W., Alba-baena N.G., Murr L.E. Explosive Shock-Wave consolidation of Aluminium powder. Carbon nanotube aggregate Mixtures: optical and electron Metallography. Metallurg. Mater. Trans., 2007. 38A(12), P. 2928-2931.

[7] Deng C.F., Wang D.Z., Zhang X.X., Ma Y.X. Damping characteristics of carbon nanotube reinforced aluminum composite. Mater. Lett., 2007, 61(14-15), P. 3229-3231.

[8] Jafari M., Abbasi M.H., Enayati M.H., Karimzadeh F. Mechanical properties of nanostructured A12024MWCNT composite prepared by optimized mechanical milling and hot pressing methods. Adv. Powder Technol., 2012, 23(2), P. 205-210.

[9] Esawi Amal M.K., Borady Mostafa A. El. Carbon nanotube-reinforced aluminium strips. Composites Sci. Technol., 2008, 68(2), P. 486-492.

[10] Morsi K., Esawi K., Borah P., Lanka S., Sayed A., Tahe M. Properties of single and dual matrix aluminumcarbon nanotube composites processed via spark plasma extrusion (SPE). Mater. Sci. Engin. A, 2010, 527(2122), P. 5686-5690.

[11] Kaminsky U.D. Practice and perspectives of implementation mechanoactivation processes in metallurgy. Nauka-Proizvodstvu, 2002, 2, P. 27-34.

[12] Yufeng Wu, Gap-Yong Kim. Carbon nanotube reinforced aluminum composite fabricated by semi-solid powder processing. J. Mater. Process. Technol. Available online 15 March 2011.

[13] He C.N., Zhao N.Q., Shi C.S., Song S.Z. Mechanical properties and microstructures of carbon nanotubereinforced Al matrix composite fabricated by in situ chemical vapor deposition. J. Alloys Compounds, 2009, 487(1-2), P. 258-262.

[14] Deng C.F., Wang D.Z., Zhang X.X., Li A.B. Processing and properties of carbon nanotubes reinforced aluminum composites. Mater. Sci. Engin. A, 2007, 444, P. 138-145.

[15] Esawi A.M.K., Morsi K., Sayed A., Abdel Gawad A., Borah P. Fabrication and properties of dispersed carbon nanotube-aluminum composites. Mater. Sci. Engin. A, 2009, 508, P. 167-173.

[16] Singhal S.K., Pasricha R., Teotia S., Kumar G., Mathur R.B. Fabrication and characterization of Al-matrix composites reinforced with amino-functionalized carbon nanotubes. Composites Sci. Technol., 2011, 72(1), P. 103-111. 